\title{
X-ray-diffraction study of crystalline Si nanocluster formation in annealed silicon-rich silicon oxides
}

\author{
D. Comedi, ${ }^{\text {a) }}$ O. H. Y. Zalloum, E. A. Irving, J. Wojcik, T. Roschuk, M. J. Flynn, and \\ P. Mascher \\ Centre for Emerging Device Technologies, Department of Engineering Physics, McMaster University, \\ Hamilton, Ontario L8S 4L7, Canada
}

(Received 2 September 2005; accepted 7 December 2005; published online 25 January 2006)

\begin{abstract}
The formation and subsequent growth of crystalline silicon nanoclusters (Si-ncs) in annealed silicon-rich silicon oxides (SRSOs) were studied by glancing angle x-ray diffraction. SRSO samples with $\mathrm{Si}$ concentrations $(y)$ of $0.40,0.42$, and 0.45 were grown by inductively coupled plasma-enhanced chemical-vapor deposition (PECVD). Samples with $y=0.42$ grown by electron-cyclotron-resonance PECVD were also studied. Annealing treatments were performed at temperatures $(T)$ of 900,1000 , and $1100{ }^{\circ} \mathrm{C}$ for times $(t)$ between 0.5 and $3 \mathrm{~h}$ in flowing Ar. As-grown SRSO films did not present signs of Si clusters (amorphous or crystalline); however, (111), (220), and (311) Bragg peaks corresponding to $c$-Si were clearly seen after annealing at $900{ }^{\circ} \mathrm{C}$ for the $y=0.45$ sample, but only barely seen for the $y=0.42$ and undetected for the $y$ $=0.40$ samples. For $T=1000{ }^{\circ} \mathrm{C}$, all studied SRSO samples clearly showed the $c$-Si diffraction peaks, which became narrower with increasing $t$ and $T$. From the width of the Si (111) peaks, the mean size of Si-ncs and their dependence on $T$ and $t$ was determined. Activation energies were deduced from the $T$ dependence by fitting the results to two growth models of Si precipitates in an $a-\mathrm{SiO}_{2}$ matrix reported in the literature. The activation energies qualitatively agree with values deduced from transmission electron microscopy studies of annealed SRSO reported in the literature. However, they are significantly lower than Si diffusion activation energies available in the literature for $\mathrm{SiO}_{2}$ with low excess $\mathrm{Si}$. A broad feature is also observed in the x-ray diffractograms for as-grown samples with low $y$, which shifts to the peak position corresponding to $a-\mathrm{SiO}_{2}$ with increasing $T$. This behavior is explained by the formation of a well-defined $a-\mathrm{SiO}_{2}$ phase with increasing $T$, where mixed $\mathrm{Si}-\mathrm{O}_{4-n} \mathrm{Si}_{n}(n=1,2,3)$ tetrahedra in the as-grown alloy are gradually converted into $\mathrm{Si}-\mathrm{O}_{4}$ and $\mathrm{Si}-\mathrm{Si}_{4}$ as phase separation of $\mathrm{Si}$ and $\mathrm{SiO}_{2}$ proceeds. From the measured $\mathrm{Si}$ (111) peak positions, small Si-ncs are found to be tensilely strained by as much as $\sim 0.8 \%$. This effect becomes insignificant as Si-ncs become larger with increasing y or T. (C) 2006 American Institute of Physics. [DOI: 10.1063/1.2162989]
\end{abstract}

\section{INTRODUCTION}

Crystalline silicon nanoclusters (Si-ncs) embedded in $\mathrm{SiO}_{2}$ form a class of materials that recently have attracted much interest as it is a heterogeneous system with appealing potential applications. One of these is the active material in Si-based light emitters for Si photonics, ${ }^{1}$ where advantage is taken of quantum confinement of charge carriers in the Si-nc to enable efficient, tunable light emission. ${ }^{2}$ In contrast to bulk $c$-Si where any luminescent process is very inefficient due to its indirect band gap, in Si-ncs the $k$ conservation rule in radiative transitions is relaxed due to the broadening of the electron and hole wave functions in $k$ space as a result of spatial confinement. The emission wavelength tunability, in turn, can be achieved by adjusting the energy separation between electronic levels through the control of the mean Si-nc size $(d)$ in the $d<100 \mathrm{~nm}$ range.

The use of $\mathrm{Si}$ nanoparticles embedded in $\mathrm{SiO}_{2}$ has been also suggested for the replacement of the monolithic floating gate of conventional flash memories in microelectronics. ${ }^{3,4}$ Such a procedure is expected to improve the immunity of

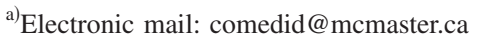

data against charge loss and therefore allow thinner tunnel oxides and smaller memory devices. Furthermore, Si-ncs have been indicated for single electron memories and spinbased quantum computation applications. ${ }^{5}$

Various methods have been used to fabricate Si-ncs embedded in a $\mathrm{SiO}_{2}$. A simple one involves the deposition of a nonstoichiometric $\mathrm{Si}$ oxide layer $\left(\mathrm{Si}_{y} \mathrm{O}_{1-y}\right)$ having a $\mathrm{Si}$ concentration $y$ above that of the stoichiometric $\mathrm{SiO}_{2}$ (i.e., $y$ $>1 / 3$ ) followed by a high-temperature heat treatment. Such a silicon-rich silicon oxide (SRSO) layer can be easily obtained by several low-temperature deposition methods, such as reactive sputtering or plasma-enhanced chemical-vapor deposition (PECVD), or by ion implantation of $\mathrm{Si}$ onto $\mathrm{SiO}_{2}$. The thermal treatment at temperatures between 900 and $1200{ }^{\circ} \mathrm{C}$ has been shown to lead to the stabilization of elemental $\mathrm{Si}$ precipitates within an amorphous $\mathrm{SiO}_{2}$ phase. ${ }^{6,7}$ The microscopic mechanism of this phase separation is still a matter of debate; however, some models have been proposed to describe Si-nc growth during annealing of SRSOs. ${ }^{8,9}$ These models assume diffusion of $\mathrm{Si}$ in the oxide to be the main rate-limiting step. Nevertheless, activation energies de- 
duced from fits to experimental data ${ }^{8-10}$ have been found to be much lower than those reported for $\mathrm{Si}$ diffusion in both crystalline and amorphous $\mathrm{SiO}_{2}$.

Another open question concerns the Si-nc formation process itself. It has been frequently assumed in the literature (and eventually observed ${ }^{11}$ ) that, in a first stage, amorphous Si precipitates are formed, which eventually crystallize when the annealing temperature is increased. In contrast, no amorphous phase could be detected under certain circumstances, ${ }^{12}$ suggesting that the Si-ncs may also grow from a crystalline seed (i.e., without an amorphous cluster precursor). This point and its connection to the process (SRSO deposition and postannealing) parameters should be very relevant for the precise control of the Si-nc size and structure required for various applications.

Due to their promising perspectives, the observation and characterization of Si-ncs in $\mathrm{SiO}_{2}$ are some of the important issues in Si nanotechnology and have been vividly discussed in the recent literature. One of the commonly used techniques is transmission electron microscopy (TEM). Dark field TEM (DFTEM) enables the acquisition of a relatively wide image of many $\mathrm{Si}$ precipitates in the amorphous $\mathrm{SiO}_{2}$ through the contrast that results from the electron diffraction signal under Bragg's condition from coherent Si planes in a Si-nc. ${ }^{7-9}$ Hence, this method allows obtaining information on the size distribution of essentially crystalline clusters. Energy-filtered TEM (EFTEM) has been used more recently to allow the separation between elemental $\mathrm{Si}$ and the $\mathrm{Si}$ in the $\mathrm{SiO}_{2}$, taking advantage of their discrimination in electron energy-loss spectra. ${ }^{10}$ The great advantage of EFTEM is that it eliminates the need for crystalline coherence and the requirement for orientation of specific Si-nc with respect to the incoming and outcoming electron beams. However, electron microscopy techniques possess a significant disadvantage related to inherent errors that originate in averaging processes, which result in poor reproducibility when analyzing different regions of the sample. An additional source of error is the tendency of underestimating the smaller clusters in the distribution since these are not always distinguishable in the images. Furthermore, this technique requires a sample preparation process that is destructive and invasive and therefore prone to interfere with the structure of the processed sample.

Powder x-ray diffraction (XRD), in contrast, presents the significant advantages of being noninvasive and nondestructive. Furthermore, the averaging process for Si-nc size determination is potentially more reliable than in TEM because the diffraction signal from a macroscopic number of clusters is averaged over the x-ray-illuminated volume. In addition, this technique can also detect amorphous precipitates as amorphous $\mathrm{Si}$ gives a characteristic x-ray diffraction pattern. ${ }^{13}$ This fact has been exploited recently to observe amorphous $\mathrm{Si}$ precipitates in $\mathrm{SiO}_{2}$ by XRD measurements. ${ }^{11}$

The purpose of this work is to study the formation and growth of Si precipitates in annealed SRSOs fabricated by inductively coupled (IC) and electron-cyclotron-resonance (ECR) PECVD. Glancing angle XRD is used and the diffraction peaks are recorded as a function of the annealing temperature and time for various Si contents in the SRSO. The results are compared to existing model predictions and to relevant results obtained by other authors from SRSO samples prepared by PECVD and ion implantation methods.

\section{EXPERIMENTAL DETAILS}

2- $\mu$ m-thick SRSO films with different $\mathrm{Si}$ contents $(y$ $=0.40,0.42$, and 0.45 in $\left.\mathrm{Si}_{y} \mathrm{O}_{1-y}\right)$ were deposited on $c$-Si wafers by IC-PECVD in a reactor built by Johnsen-Ultravac of Burlington, Ontario. The substrate temperature during deposition was fixed at $120^{\circ} \mathrm{C}$. Some reference SRSO samples with $y=0.42$ were also grown by ECR-PECVD. The $y$ values were determined by Rutherford backscattering (RBS) measurements for samples prepared on both $c$-Si and glassy carbon substrates. After growth, wafers were cleaved and annealed in a quartz tube furnace under flowing Ar for times $(t)$ varying between $0.5-3 \mathrm{~h}$ and temperatures $(T)$ of 900,1000 , and $1100{ }^{\circ} \mathrm{C}$. Each sample was annealed only once at a specific $T$ and a certain $t$, i.e., no cumulative annealing experiments were performed.

$\mathrm{X}$-ray diffraction measurements were carried out with the $\mathrm{Cu} K \alpha$ line in a Bede D1 diffractometer. A silicon crystal monochromator was used to eliminate $K \beta$ contributions from the $\mathrm{Cu} x$-ray source. A fixed glancing incidence angle $(\omega$ $=2.5^{\circ}$ ) was used in order to fix and increase the SRSO film depth probed by the x-ray beam as compared to conventional $\theta-2 \theta$ scans, while $2 \theta$ was scanned typically between $10^{\circ}$ and $60^{\circ}$ at $0.075^{\circ}$ steps. The incoming $\mathrm{x}$-ray beam was collimated to a width of $0.5 \mathrm{~mm}$ so that illuminated areas were smaller than the sample size of typically $2 \mathrm{~cm}^{2}$. The angular acceptance of the detector was limited to $0.07^{\circ}$ by a $0.5 \mathrm{~mm}$ wide slit. Acquisition times per angular step of $300-500 \mathrm{~s}$ were used.

It is well known that as a diffracting crystallographically coherent region becomes spatially smaller, the Bragg peaks are broadened. The volume average size of the diffracting region $(d)$ can be related to the full width at half maximum $B$ of a Bragg peak in a $2 \theta$ scale through the Scherrer formula: ${ }^{14}$

$$
d=\frac{K \lambda}{B \cos \theta},
$$

where $\lambda$ is the $\mathrm{x}$-ray wavelength $(0.1541 \mathrm{~nm}), \theta$ is the Bragg angle, and $K$ is a constant on order of unity whose exact value depends on the specific shape and size distribution of the crystalline clusters and on the specific crystallographic direction of the diffracting planes. ${ }^{14}$ Calculated values for the (111) direction in many different shapes and structures are close to $K=0.9$ to within few percent, ${ }^{14}$ so we have consistently adopted this value for the $\mathrm{Si}$ (111) reflection.

The assumptions concerning the use of XRD and the Scherrer formula (a method hereafter referred to as XRD-SF) to deduce the Si-nc size will be thoroughly discussed in Sec. IV. However, this method has been found to yield full agreement with the size values deduced by TEM in nanocrystalline materials within the experimental uncertainties of both techniques. ${ }^{15}$ Furthermore, several research groups have independently demonstrated good overall agreement between mean particle size determinations by XRD-SF and TEM in various nanoparticle systems ${ }^{16-18}$ within estimated error bars. 


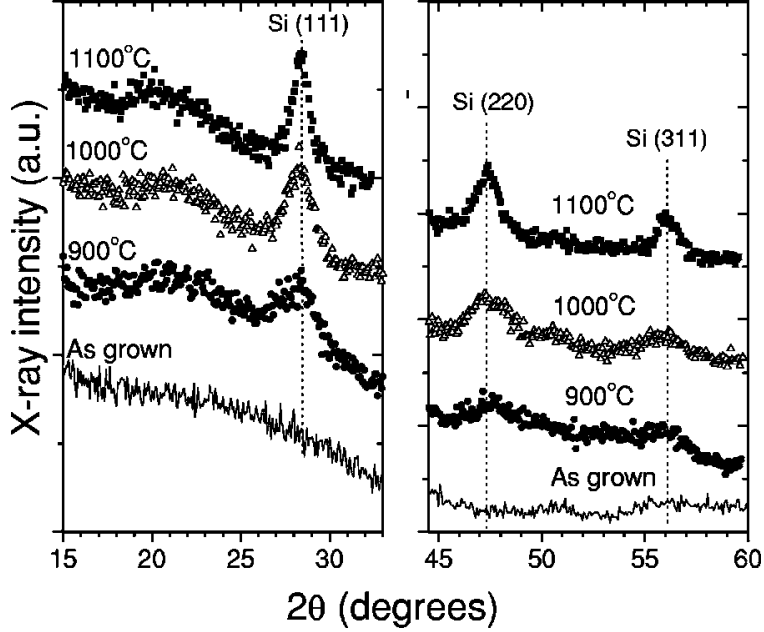

FIG. 1. X-ray diffractograms for $\mathrm{Si}_{y} \mathrm{O}_{1-y}$ samples with $y=0.45$ grown by IC-PECVD and annealed at different $T$ for $2 \mathrm{~h}$. The diffractogram for an unannealed sample is also shown as a reference. The vertical dotted lines indicate the expected positions of the Si (111), (220), and (311) Bragg peaks. The data sets are shifted vertically for clarity.

The FWHMs of the diffraction peaks were corrected for the instrumental FWHM of $0.09^{\circ}$ as measured for a polycrystalline powder Si sample before introducing them into Eq. (1) to determine $d$.

A few SRSO samples grown by either IC-PECVD or ECR-PECVD were examined comparatively using XRD and photoluminescence (PL) measurements. Both types of samples exhibited qualitatively similar behavior. As will be shown below, the results reported here for IC-PECVD samples are qualitatively similar to those reported previously for ECR-PECVD films. ${ }^{6}$ The PL results for the films studied here can be correlated with the XRD results and will be presented in a forthcoming publication.

\section{RESULTS}

Figure 1 shows the x-ray diffractograms for $\mathrm{Si}_{y} \mathrm{O}_{1-y}$ samples with $y=0.45$ grown by IC-PECVD for various annealing temperatures $T$. It can be seen that the data for the as-grown material do not show any clear features. For annealing at $T=900{ }^{\circ} \mathrm{C}$, in contrast, four peaks can be resolved at $\sim 22.0^{\circ}, 28.3^{\circ}, 47.5^{\circ}$, and $55.9^{\circ}$. The first peak is very broad and it has been attributed to an amorphous $\mathrm{SiO}_{2}$ phase. ${ }^{19-21}$ The other three peaks are very close to the expected (111), (220), and (311) Bragg peaks of Si (whose positions are shown in the figure as dotted vertical lines). The results in Fig. 1 demonstrate that crystalline Si precipitates are formed within an amorphous $\mathrm{SiO}_{2}$ matrix during the annealing at $900{ }^{\circ} \mathrm{C}$. This result is in qualitative agreement with previous analysis by infrared spectroscopy and ellipsometry of similar samples grown by ECR-PECVD. ${ }^{6}$ As can also be seen in Fig. 1, for $T=1000$ and $1100{ }^{\circ} \mathrm{C}$, the Si peaks become narrower, indicating that the average size of Si-ncs increases with increasing $T$.

Figure 2 shows the $\mathrm{x}$-ray diffraction pattern in the $\mathrm{Si}$ (111) region for the $y=0.42$ sample deposited by IC-PECVD. As in Fig. 1, the broad feature due to $a-\mathrm{SiO}_{2}$ can be seen close to $22^{\circ}$ for all three annealed samples. It is interesting to

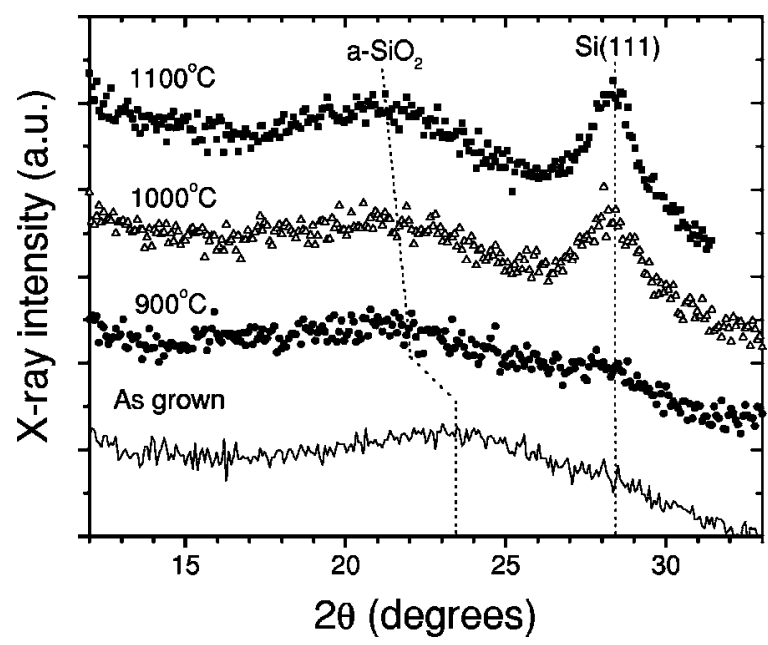

FIG. 2. X-ray diffractograms in the region of the $a-\mathrm{SiO}_{2}$ and $\mathrm{Si}$ (111) peaks for $\mathrm{Si}_{y} \mathrm{O}_{1-y}$ samples with $y=0.42$ grown by IC-PECVD and annealed at different $T$ for $2 \mathrm{~h}$. The diffractogram for an unannealed sample is also shown as a reference. The vertical dotted line at $28.4^{\circ}$ indicates the expected position of the Si (111) Bragg peak. The almost vertical dotted lines between $21.2^{\circ}$ and $23.4^{\circ}$ are guide to the eye and are meant to show a shift of the $a-\mathrm{SiO}_{2}$-like peak to lower $2 \theta$ that occurs as $T$ is increased. The data sets are shifted vertically for clarity.

note that the as-grown film also shows a broad feature, however, shifted to a larger $2 \theta$ value. Note that this feature is not as clearly observed in the as-grown $y=0.45$ material (Fig. 1). This is evidence that oxide domains having some mediumrange order can already form in as-grown material; however, their formation is inhibited as $y$ is increased to $y=0.45 .{ }^{21}$ As can also be appreciated from Fig. 2, the $a$ - $\mathrm{SiO}_{2}$-like peak shifts to lower $2 \theta$ as $T$ is increased. In addition, a trace of the Si (111) peak just starts to develop at $900{ }^{\circ} \mathrm{C}$, while it becomes much more defined at $1000{ }^{\circ} \mathrm{C}$ and narrower at $1100{ }^{\circ} \mathrm{C}$, indicating the formation and growth of the Si precipitates.

Figure 3 shows the XRD data obtained for $y=0.40$. In this case the $\mathrm{Si}$ (111), (220), and (311) peaks are small but detectable after annealing at 1000 and $1100{ }^{\circ} \mathrm{C}$, but are ab-

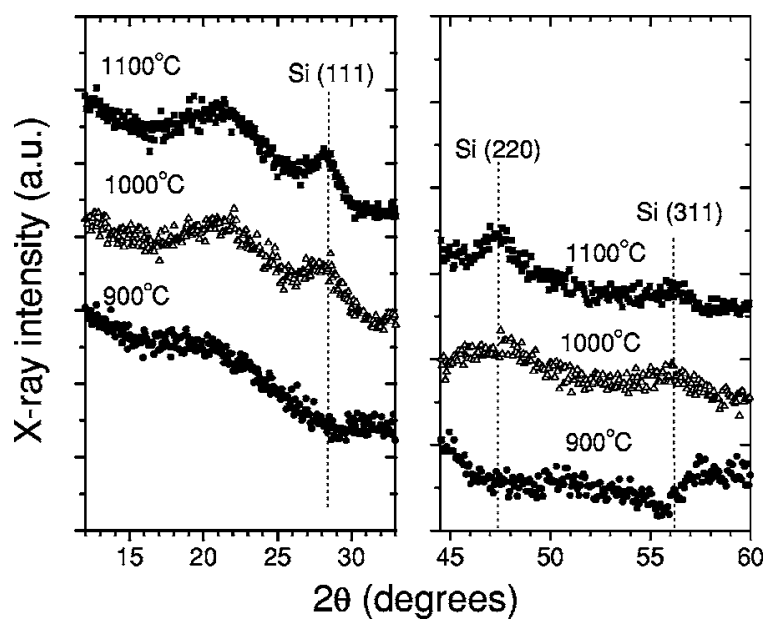

FIG. 3. X-ray diffractograms for $\mathrm{Si}_{y} \mathrm{O}_{1-y}$ samples with $y=0.40$ grown by IC-PECVD and annealed at different $T$ for $2 \mathrm{~h}$. The vertical dotted lines indicate the expected positions of the Si (111), (220), and (311) Bragg peaks. Data sets are shifted vertically for clarity. 


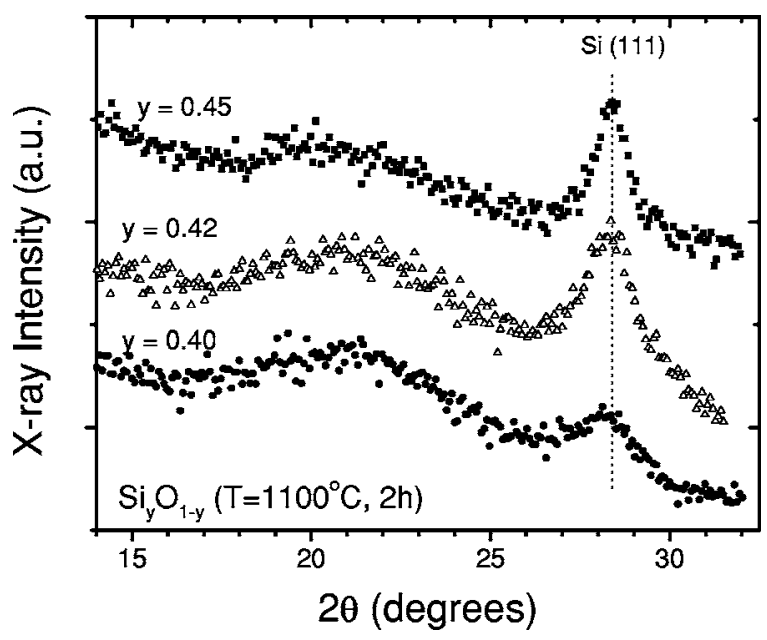

FIG. 4. X-ray diffractograms for $\mathrm{Si}_{y} \mathrm{O}_{1-y}$ samples with $y=0.40,0.42$, and 0.45 grown by IC-PECVD and annealed at $T=1100{ }^{\circ} \mathrm{C}$ for $2 \mathrm{~h}$. The vertical dotted line indicates the expected position of the Si (111) Bragg peak. Data sets are shifted vertically for clarity.

sent in the diffractograms obtained for the sample annealed at $900{ }^{\circ} \mathrm{C}$. Again, one can see that the Si peaks become narrower as $T$ is increased.

Figure 4 summarizes the behavior of the $\mathrm{Si}$ (111) peak with increasing $\mathrm{Si}$ content in the SRSO while keeping the annealing parameters identical $\left(T=1100{ }^{\circ} \mathrm{C}, t=2 \mathrm{~h}\right)$. It can be seen that the peak becomes narrower as the $\mathrm{Si}$ content increases. This is evidence that the mean size of crystalline $\mathrm{Si}$ regions increases with increasing excess $\mathrm{Si}$ at this $T$.

For some of the samples, the evolution of the diffraction peaks with annealing time at a fixed $T=1100{ }^{\circ} \mathrm{C}$ was recorded. The peaks become narrower with increasing time (not shown), indicating that the Si crystalline regions become slightly larger with increasing $t$.

\section{DISCUSSION}

\section{A. Analysis of the $a-\mathrm{SiO}_{2}$ and $\mathrm{Si}$ (111) peaks}

Figure 5 shows the diffractogram after background correction in the region of the $a-\mathrm{SiO}_{2}$-like and $\mathrm{Si}$ (111) peaks for a sample that exhibits a relatively weak Si peak $(y=0.40)$. The background correction was done by adjusting and subtracting the diffractogram obtained in the same $2 \theta$ region for a $c$-Si sample cleaved from the same wafers as for the depositions. It can be seen from Fig. 5 that the XRD pattern can be satisfactorily fit by the sum of two Lorentzians, one corresponding to the $a-\mathrm{SiO}_{2}$-like peak and the other for the $\mathrm{Si}$ (111) peak. This procedure was systematically applied to all the XRD curves obtained and enabled us to consistently determine the peak positions, widths, and intensities.

Figure 6(a) shows the $\mathrm{Si}$ (111) peak position as a function of the annealing temperature as determined for all the samples annealed for $2 \mathrm{~h}$. It can be seen that the peak is shifted towards low angles with respect to the position observed for the $\mathrm{Si}$ (111) in polycrystalline $\mathrm{Si}$ [also shown in Fig. 6(a) as a horizontal dotted line]. This shift is larger for samples with lower Si concentrations and annealed at lower annealing temperatures, i.e., for conditions where smaller precipitates are formed. This result indicates that small $\mathrm{Si}$

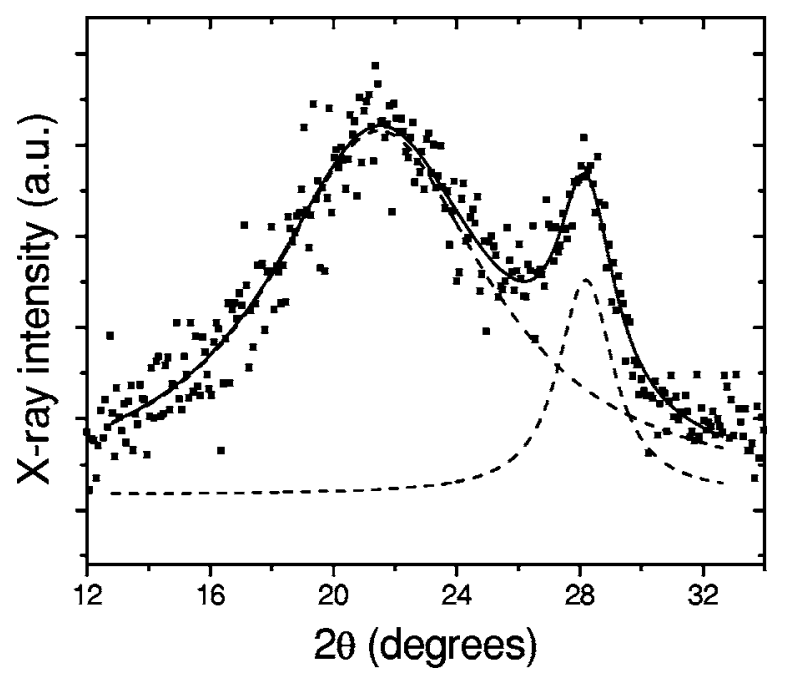

FIG. 5. Diffractogram for a $\mathrm{Si}_{y} \mathrm{O}_{1-y}$ film with $y=0.40$ annealed at $1100{ }^{\circ} \mathrm{C}$ for $2 \mathrm{~h}$ (from Fig. 4) after background correction. Also shown are the results of the least-squares fit of the sum of two Lorentzians to the experimental data corresponding to the $a-\mathrm{SiO}_{2}$-like and $\mathrm{Si}(111)$ peaks.

clusters are uniformly strained in tension, and that this effect is reduced as the clusters grow. The strain in the small Si-ncs amounts to about $0.3 \%-0.8 \%$, as can be seen with the help of the strain scale in Fig. 6(a). This scale has been calculated in terms of the $\mathrm{Si}-\mathrm{Si}$ bond-length variation with respect to bulk Si using the corresponding $2 \theta$ values and Bragg law. We attribute the observed strain to the stretching of $\mathrm{Si}-\mathrm{Si}$ bonds with respect to the bulk value of $2.35 \AA$ occurring near the Si-nc/oxide interface due to the shorter $\mathrm{Si}-\mathrm{O}$ bonds $[\sim 1.6 \AA$ (Ref. 22)]. This effect becomes less significant as more $\mathrm{Si}$ atoms incorporate to the $\mathrm{Si}$ clusters and contribute to the growth of an unperturbed Si core.

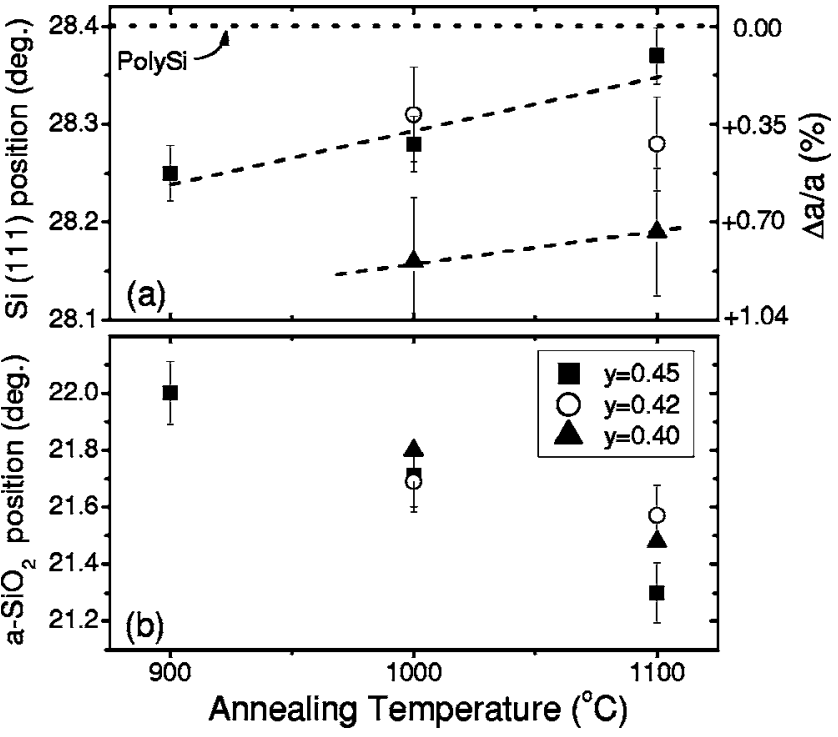

FIG. 6. Position of the (a) $\mathrm{Si}$ (111) and (b) a- $\mathrm{SiO}_{2}$-like peaks in a $2 \theta$ scale as deduced from a least-squares fit to the XRD data for $\mathrm{Si}_{y} \mathrm{O}_{1-y}$ samples grown by IC-PECVD $(y=0.40,0.42$, and 0.45$)$ as a function of the annealing temperature. The strain scale $(\Delta a / a)$ in the right-hand vertical axis of (a) was calculated from corresponding $2 \theta$ values using Bragg law. The dashed straight lines in (a) are meant to guide the eye only, while the horizontal dotted line at $2 \theta=28.40^{\circ}$ is the position of the $\mathrm{Si}(111)$ peak in polycrystalline $\mathrm{Si}$ which is assumed to be unstrained. 
Figure 6(b) shows the $a-\mathrm{SiO}_{2}$ peak position as a function of the annealing temperature. In this case, the peaks shift to lower angles as the annealing temperature is increased. It is interesting to note that a shift of the $a-\mathrm{SiO}_{2}$-like peak with increasing annealing temperature has been observed before in XRD studies of thermally grown $\mathrm{Si}_{y} \mathrm{O}_{1-y}$ films, but no interpretation for this effect was given. ${ }^{19}$ The diffraction peak at low $2 \theta$ observed in XRD patterns from amorphous films is referred to in the literature as "first scattering peak" (FSP). It has been related to atomic fluctuations ["quasiBragg planes" (Ref. 20)] with characteristic correlation lengths of about $\sim 1 \mathrm{~nm}$ resulting from "medium-range order" ${ }^{, 13,20}$ in the amorphous lattice. For the case of $a-\mathrm{SiO}_{2}$, the peak at $\sim 21.5^{\circ}$ mimics $^{20}$ the reflection from $\{111\}$ planes to $\beta$-cristobalite quartz (period of $0.411 \mathrm{~nm}$ ). A similar FSP peak is observed ${ }^{13}$ for amorphous $\mathrm{Si}$ at $\sim 28.5^{\circ}$, which mimics the Si (111) peak of crystalline Si (period of $0.313 \mathrm{~nm}$ ). Hence, we interpret the shift of the FSP peak to lower angles depicted in Fig. 6(b) as being due to an increase of the average period of quasi-Bragg planes that occurs during spinodal decomposition of SRSO ${ }^{21}$ One can imagine the process as a gradual transition from $\mathrm{Si}-\mathrm{O}_{4-n} \mathrm{Si}_{n}(n=1,2,3)$ mixed tetrahedra to pure $\mathrm{Si}-\mathrm{O}_{4}$ and $\mathrm{Si}-\mathrm{Si}_{4}$ tetrahedra that form the basic atomic elements of stoichiometric $\mathrm{SiO}_{2}$ and elemental $\mathrm{Si}$, respectively. Such an alloy regime with oxidation states of $\mathrm{Si}$ lower than +4 characterizes the $\mathrm{Si} / \mathrm{SiO}_{2}$ planar interface, ${ }^{23}$ and the transition from it into $\mathrm{Si}-\mathrm{O}_{4}$ and $\mathrm{Si}_{-}-\mathrm{Si}_{4}$ has indeed been observed in SRSO by high-resolution photoelectron spectroscopy. ${ }^{24}$

\section{B. The determination of $d$ by XRD-SF}

The use of the XRD-SF method to determine $d$ [Eq. (1)] involves some intrinsic assumptions that are often overlooked and not properly discussed in the literature. The first one is that the broadening of the diffraction peak is entirely due to the size effect, i.e., nonuniform strain effects are negligible. This assumption is well justified by the fact that the $\mathrm{Si}$ precipitates are expected to be in the nanometer scale due to the small amount of excess $\mathrm{Si}$ in the SRSO. Hence a large size broadening effect is expected particularly for crystalline regions in this case. In addition, since these crystalline precipitates are formed in an amorphous $\mathrm{SiO}_{2}$ matrix following a high-temperature thermal treatment, a large fraction of the strain is expected to relax by bond reorganization in the amorphous matrix side of the cluster/oxide interface. ${ }^{25}$ Furthermore, nonuniform strain in crystallites is usually associated with the presence of extended defects, ${ }^{26}$ such as dislocations, which are highly unlikely in a nanocrystal embedded in an amorphous matrix. Since strain broadening, if any, is expected to increase faster with increasing diffraction angle than size broadening ${ }^{27}$ its effects should be minimized when considering Bragg peaks at lower angles. Due to this, we have chosen here to focus our quantitative analysis on the $\mathrm{Si}$ (111) peak, which has the additional advantage of being more intense and better defined than the (220) and (311) peaks due to its larger structure factor. ${ }^{28}$ In any case, as can be appreciated by inspection of Figs. 1 and 3, the (220) and (311) peaks qualitatively follow the trends observed for the (111) peak.

Another implicit assumption in the use of Eq. (1) is that local coherence (i.e., partial crystallographic coherence between neighboring crystalline clusters) is negligible. ${ }^{29}$ This assumption is also justified by the fact that the Si clusters are embedded in an amorphous matrix. As pointed out in Ref. 29 , in contrast to nanocrystalline materials where local partial coherence between adjacent crystallites is quite possible, such an effect should be very small or absent when the crystalline clusters are separated by amorphous regions.

\section{The association of $d$ with the mean size of Si precipitates}

It is interesting to note that we do not observe in the XRD patterns any signature of amorphous clusters or amorphous regions in $\mathrm{Si}$ precipitates, in contrast to others. ${ }^{11} \mathrm{~A}$ possible reason for this is that the volume associated with amorphous regions is small in our case. EFTEM and highresolution TEM (HRTEM) studies in annealed SRSOs have shown the presence of amorphous $\mathrm{Si}$ precipitates that consisted of a crystalline core and an amorphous shell. ${ }^{10} \mathrm{~A}$ comparison between conventional TEM and EFTEM in those films indicated that the mean Si-precipitate size was systematically larger by $25 \%$ than the mean size of the Si crystalline regions. Although such a size difference was shown to be on the same order of the quoted error bars, it would correspond to a fractional difference in volume of about $75 \%$. Such a difference, in turn, could imply in amorphous $\mathrm{Si}$ regions large enough to yield observable contributions to the $\mathrm{x}$-ray curves. The fact that we do not observe any indicates that either the amorphous regions in our samples, if any, are relatively small or strongly disordered. With this in mind, it will be assumed in what follows that the $d$ values deduced here are proportional to the actual mean Si-precipitate sizes, and that the difference between these is probably not more than $\sim 20 \%$.

The uncertainties attributed to $d$ throughout this work (shown as error bars in the figures) are statistical errors associated with the determination of the Si (111) Bragg peak FWHM only. That is, we do not consider systematic errors that could result from possible inaccuracies in the underlying assumptions made. In any case, all discussions below involve essentially the relative variations of the mean size of Si-ncs, whose accuracy should be well represented by the error bars of $d$.

\section{Dependence of $d$ on annealing time and temperature}

The growth of Si precipitates with annealing time and temperatures has been described by a simple model that considers diffusion of $\mathrm{Si}$ from bulk $\mathrm{Si}_{y} \mathrm{O}_{1-y}$ towards a $\mathrm{Si}$-cluster seed. ${ }^{8}$ This model has been used often to explain Si-nc formation in $\mathrm{SRSO}^{30-32}$ and even considered as a reliable method to obtain Si diffusion coefficients in $a-\mathrm{SiO}_{2} \cdot{ }^{33} \mathrm{How}-$ ever, we point out that it should be considered with great care, as it involves many unknown parameters, such as the 


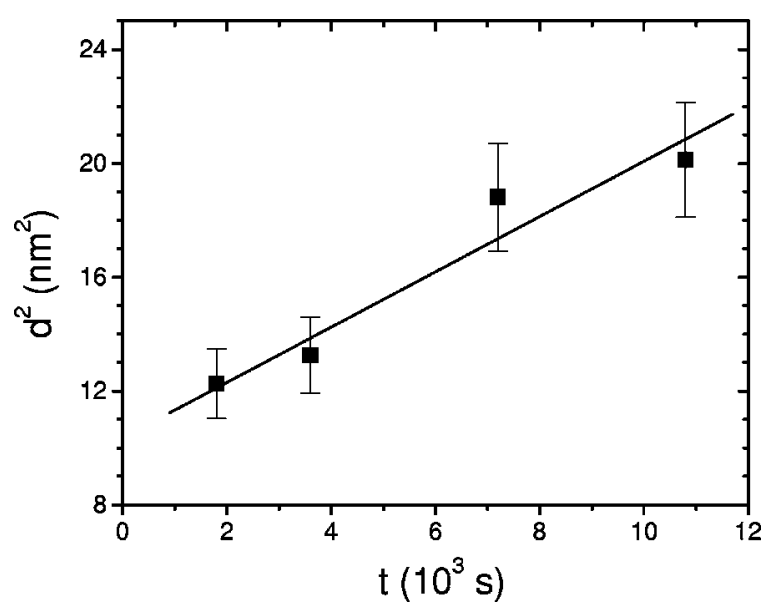

FIG. 7. The mean Si-nc size as estimated from the XRD data and Eq. (1) squared $\left(d^{2}\right)$, as a function of annealing time, for $\mathrm{Si}_{y} \mathrm{O}_{1-y}$ samples with $y$ $=0.42$ grown by ECR-PECVD and annealed at $1100{ }^{\circ} \mathrm{C}$. The straight line is a least-squares fit to the data.

initial seed size, the $\mathrm{Si}$ concentration at the immediate $\mathrm{Si}$ precipitate/oxide interface, and the $\mathrm{Si}$ concentration profile between this interface and the $\mathrm{Si}_{y} \mathrm{O}_{1-y}$ "bulk" (i.e., a region where no Si precipitation exists and the Si concentration can be assumed to be equal to its mean value $y$ ). Furthermore, it considers only diffusion; it ignores nucleation or crystallization barriers and does not consider changes in the Si concentration profile with increasing annealing time. Nevertheless, using a set of reasonable assumptions, Nesbit ${ }^{8}$ was able to obtain self-consistent diffusion coefficients from his model by adjusting it to the mean $\mathrm{Si}$-cluster radius as determined from TEM measurements. By assuming spherical symmetry and a linear concentration gradient between the $\mathrm{Si}$ cluster/ oxide immediate interface and the $\mathrm{Si}_{y} \mathrm{O}_{1-y}$ bulk, he obtained

$$
r^{2}-r_{i}^{2}=2 t\left(\frac{y-C_{\mathrm{INT}}^{\mathrm{Si}}}{1-C_{\mathrm{INT}}^{\mathrm{Si}}}\right) D^{*}
$$

where $r$ and $r_{i}$ are the final and initial $\mathrm{Si}$-nc radii, respectively, $t$ is the annealing time, $C_{\mathrm{INT}}^{\mathrm{Si}}$ is the $\mathrm{Si}$ concentration at the immediate $\mathrm{Si}$-nc/oxide interface, and $D^{*}$ is the $\mathrm{Si}$ diffusion coefficient of the $\mathrm{Si}$ atom across the concentration gradient at the annealing temperature $T$. In other words, the Si-precipitate size squared should be proportional to $t$ and $D^{*}$. For $D^{*}$, in turn, an activated behavior,

$$
D^{*}=D_{0}^{*} \exp \left(-E_{a} / k T\right)
$$

is expected, where $D_{0}^{*}$ is a diffusivity prefactor, $E_{a}$ is the diffusion activation energy, and $k$ is the Boltzmann constant.

Making the assumption that $d \propto r$, we have plotted $d^{2}$ in Figs. 7 and 8 as a function of $t$ and an Arrhenius plot for $d^{2}$ obtained for various SRSO samples, respectively. From Fig. 7 , it can be seen that $d^{2}$ indeed increases with $t$ in consistency with the model, although we should mention that the available data and error bars do not completely rule out a dependence other than the linear. From the Arrhenius plot (Fig. 8) we obtain activation energies of $1.8 \mathrm{eV}$ for samples with $y=0.42$ and $1.3 \mathrm{eV}$ for $y=0.45$. The values are close to the one found by Nesbit himself ${ }^{8}(1.9 \mathrm{eV})$ and slightly larger than that found by Iacona et al. ${ }^{10}(1.2 \mathrm{eV})$ in their diffusional

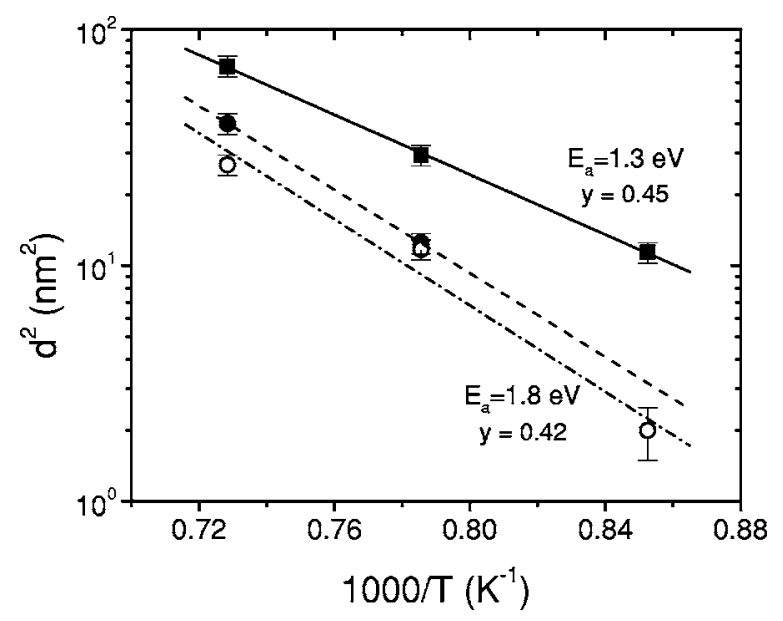

FIG. 8. The mean Si-nc size as estimated from the XRD data and Eq. (1) squared $\left(d^{2}\right)$, in a logarithmic scale, as a function of the reciprocal annealing temperature. The data correspond to the following $\mathrm{Si}_{y} \mathrm{O}_{1-y}$ samples. ICPECVD: $y=0.45$ (solid squares) and $y=0.42$ (solid circles); ECR-PECVD: $y=0.42$ (open circles). The lines are least-squares fits to the data assuming an Arrhenius behavior; the corresponding deduced activation energies are also shown.

analysis of TEM data on Si-precipitate growth. As noted already in both references, these values are much lower than the diffusion activation energies measured in diffusion experiments on crystalline quartz ${ }^{34}(7.6 \mathrm{eV})$, amorphous fused ${ }^{35} \mathrm{SiO}_{2}(6 \mathrm{eV})$ and $\mathrm{Si}$-ion-implanted thermally grown $\mathrm{SiO}_{2}$ [4.74 eV (Ref. 33); $5.2 \mathrm{eV}$ (Ref. 36)]. It should be pointed out, however, that the specific power dependence of $D^{*}$ on $r$ [Eq. (2)], and therefore the specific $E_{a}$ values obtained from the model, depend on the particular Si concentration profile assumed in the calculation. Nevertheless, the discrepancy between these and the diffusion activation energies is so large that it is doubtful that it could be explained by any other reasonable assumed $\mathrm{Si}$ concentration profile.

A more sophisticated approach to describe Si-precipitate growth assumes that all the $\mathrm{Si}$ atoms are already nucleated in small clusters in the as-deposited film and that the larger $\mathrm{Si}$ clusters grow due the capture of $\mathrm{Si}$ atoms released from the smaller clusters through the oxide ${ }^{9}$ (diffusion-limited Ostwald ripening theory ${ }^{37}$ ). The driving force for this process is the minimization of the interfacial energy which is proportional to the interface area. ${ }^{9}$ The predictions of this model are (1) the mean cluster size should increase with increasing annealing time and temperature as the larger clusters grow while the smaller ones gradually shrink and eventually disappear, (2) the cluster density increases with increasing $y$ but decreases with increasing $T$ and $t$, and (3) the mean size does not depend on the mean $\mathrm{Si}$ concentration as long as the distance between clusters is large as compared to the clusters size. Prediction (1) is consistent with our observations. We do not have clear evidence against or confirming prediction (2). Prediction (3), however, does not agree with actual measurements: this can be seen from Fig. 4 which, as previous work, ${ }^{8,10}$ shows that the mean Si-cluster size increases with the excess Si concentration. Bonafos et al. ${ }^{9}$ showed that this discrepancy can be accounted for indirectly by including an interaction between the diffusion fields associated to individual clusters. This interaction increases with increasing ex- 


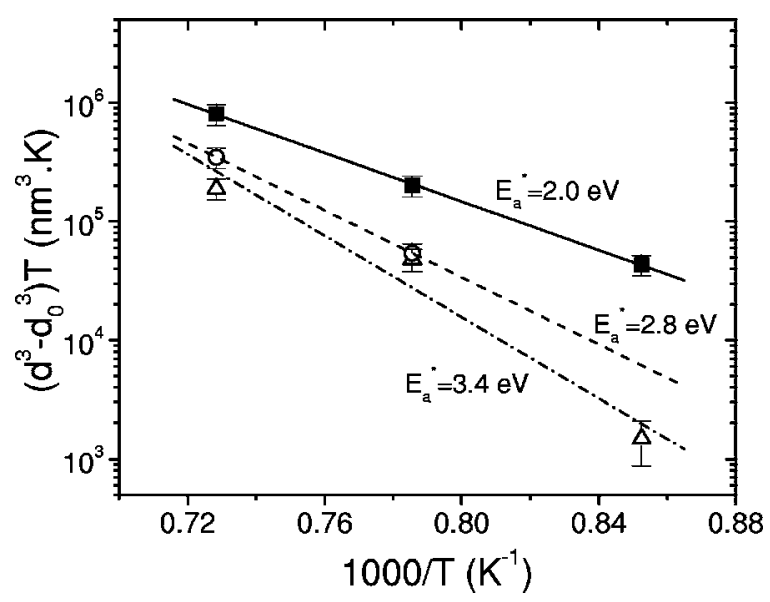

FIG. 9. $\left(d^{3}-d_{0}^{3}\right) \mathrm{T}$ in a logarithmic scale as a function of the reciprocal annealing temperature for the same $\mathrm{Si}_{y} \mathrm{O}_{1-y}$ samples as in Fig. 8. Here, $d$ is the mean Si-nc size as estimated from the $\mathrm{Si}$ (111) peak and Eq. (1), $d_{0}$ $=0.116 \mathrm{~nm}$, and $T$ is the annealing temperature in Kelvin. The lines are least-squares fits to the data assuming an Arrhenius behavior; the corresponding deduced activation energies are also shown.

cess $\mathrm{Si}$ concentration because of the increasing density of (and therefore decreasing distance between) Si clusters, and leads to an overall increase of the cluster size with increasing $y$.

A quantitative measure of the increase of the mean cluster radius with time and temperature is derived as ${ }^{9}$

$$
\bar{r}^{3}=\frac{C t}{T} \exp \left(-E_{a}^{*} / k T\right)+\bar{r}_{0}^{3},
$$

where $C$ is a constant, $r_{0}$ is a critical radius for Si nucleation, and $E_{a}^{*}$ is an activation energy. Therefore, if we assume $d$ $\approx 2 \bar{r}$ and define $d_{o}=2 \bar{r}_{0}$, an Arrhenius plot for $\left(d^{3}-d_{0}^{3}\right) T$ should yield a straight line. Such a plot is shown in Fig. 9 where, as in previous work, ${ }^{10}$ a value of $0.58 \mathrm{~nm}$ (Ref. 38) has been assumed for $\bar{r}_{0}$.

By definition, the so obtained $E_{a}^{*}$ values should be the sum of the binding energy is necessary to extract one atom from a $\mathrm{Si}$ precipitate, and a diffusion activation energy is needed to bring this atom to another precipitate. ${ }^{9}$ As can be seen in Fig. 9, the values obtained in the present work are in the 2.0-3.4 eV range. They are indeed larger than those obtained from the purely diffusional model above (Fig. 8) due to the inclusion of the binding energy. Furthermore, they are close to those deduced by Bonafos et al. ${ }^{9}(2.8 \mathrm{eV})$ and Iacona et al. ${ }^{10}(1.5-2.1 \mathrm{eV})$ in applying the same model to their own TEM data on Si nanocluster growth in annealed SRSO. However, they are still significantly lower than the diffusion energies deduced from Si self-diffusion experiments in crystalline and amorphous $\mathrm{SiO}_{2}(4.34-7.6 \mathrm{eV})$.

A possible reason for the discrepancies could be enhanced diffusion in SRSOs related to the large excess $\mathrm{Si}$ typically needed for Si clustering to occur at observable levels. It is reasonable that during the phase-separation process into $\mathrm{SiO}_{2}$ and $\mathrm{Si}$ domains, intermediate amorphous suboxide regions could exist where some of the excess $\mathrm{Si}$ could be coordinated to only two or three $\mathrm{O}$ neighbors (instead of four as in stoichiometric $\mathrm{SiO}_{2}$ ) leaving one or two unpaired $\mathrm{Si}$ bonds (i.e., O vacancies). Such undercoordinated excess $\mathrm{Si}$ atoms could be fast diffusers due to the lower-energy cost required to release them from the lattice. Excess Si could be also accommodated as interstitials, however, these are not expected to have low diffusion barriers. Diffusion experiments in $\mathrm{SiO}_{2}$ with some $\mathrm{Si}$ excess have been performed in the past, ${ }^{33}$ however, the reported activation energy $(4.74 \mathrm{eV})$ is still high as compared to those deduced for SRSO. Nevertheless, it must be realized that the excess Si content considered in Ref. 33 was considerably lower than those in the samples used in the TEM studies ${ }^{8-10}$ and in the present XRD study of Si-cluster growth in annealed SRSO. Diffusion experiments in SRSO having larger Si excess concentrations using $\mathrm{Si}$ isotopes should be useful to clarify this point.

Low diffusion barriers could also be associated with the strain and compositional interfaces present in the materials studied here. It is known that strain and other effects at surfaces can dramatically reduce diffusion barriers. For example, diffusion of Ge down through the top four layers of $\mathrm{Si}$ has been found to have diffusion barriers of only about half that of the bulk. ${ }^{39}$ The effect has been attributed to defectmediated diffusion and the particularly low defect formation energies at the Si surface. Clearly, similar effects could also occur at internal surfaces, such as the Si-nc/Si oxide interfaces present in the systems studied here.

An alternative reason for the low activation energies obtained here and in Refs. 8-10 as compared to the reported $\mathrm{Si}$ diffusion activation energies could be related to some of the simplifying assumptions made in the diffusion ${ }^{8}$ and Ostwald ripening ${ }^{9}$ models. For instance, both ignore spinodal decomposition as a driving factor for the phase-separation process. Spinodal decomposition could involve the cooperative motion of several atoms and not just $\mathrm{Si}$ diffusion in a rigid lattice, which in turn could involve lower effective activation energies. Atomic exchanges driven by the minimization of the internal energy have been considered in binary metal systems within spinodal decomposition models, such as the dynamic Ising model. ${ }^{40}$ The extension of this or of similar treatments appears to be needed to satisfactorily describe the $\mathrm{Si} / \mathrm{SiO}_{2}$ phase-separation process during high-temperature annealing of $a-\mathrm{Si}_{y} \mathrm{O}_{1-y}$ films.

\section{CONCLUSIONS}

The formation and growth of Si-ncs in an annealed SRSO with various Si contents deposited by IC-PECVD and by ECR-PECVD were studied by XRD. The following conclusions were drawn.

(1) As-grown SRSO films do not show signs of Si precipitates.

(2) Diffractograms for as-grown SRSO films with $y=0.40$ and $y=0.42$ exhibit a broad feature centered at $2 \theta$ $\sim 23^{\circ}$, which shifts to the expected value ${ }^{20}$ for $a-\mathrm{SiO}_{2}$ of $\sim 21.5^{\circ}$ with increasing annealing. This behavior is interpreted as a signature of the formation of a welldefined $a-\mathrm{SiO}_{2}$ phase by spinodal decomposition with increasing $T$. The assumption that mixed $\mathrm{Si}-\mathrm{O}_{4-n} \mathrm{Si}_{n}$ tetrahedral units are gradually converted into $\mathrm{Si}-\mathrm{O}_{4}$ and $\mathrm{Si}_{-} \mathrm{Si}_{4}$ tetrahedra is consistent with the present XRD data. $^{21}$ 
(3) No evidence for amorphous Si precipitates was found in any of the studied samples, in contrast with recent XRD studies of vacuum-annealed SRSO films ${ }^{11}$ and TEM studies. ${ }^{10}$

(4) XRD measurements of SRSO films with $y=0.45$ annealed at $900{ }^{\circ} \mathrm{C}$ show a clear evidence for Si-nc formation. No sign for Si-nc is found for $y=0.40$ within the present detection limit at this $T$, but it is observed at $1000{ }^{\circ} \mathrm{C}$.

(5) Once formed, Si-ncs grow with increasing $T$ and $t$. From the dependence of $d$ on $T$, activation energies were determined using a diffusion model ${ }^{8}$ and a model based on Ostwald ripening theory. ${ }^{9}$ The activation energies qualitatively agree with the values previously determined by TEM measurements and the same models in SRSO samples prepared by PECVD (Refs. 8 and 10) and Si ion implantation into $\mathrm{SiO}_{2} .{ }^{9}$ However, they are significantly lower than diffusion activation energies available in the literature for $\mathrm{SiO}_{2}$ with excess $\mathrm{Si}^{33}$

(6) Small Si-ncs are strained tensilely by values on the order of $0.5 \%$, as deduced from the shift of the Si (111) peak with respect to the value observed in bulk Si. This effect disappears in large Si-ncs.

\section{ACKNOWLEDGMENTS}

The authors gratefully acknowledge G. Pearson for assistance with the XRD measurements and J. Garrett for help with the annealing furnace. This work has been funded by the Ontario Research and Development Challenge Fund (ORDCF) under the Ontario Photonics Consortium (OPC) and by Ontario Centres of Excellence (OCE) Inc.

${ }^{1}$ Silicon Photonics, edited by L. Pavesi and D. J. Lockwood (Springer, Berlin, 2004).

${ }^{2}$ R. J. Walters, G. I. Bourianoff, and H. A. Atwater, Nat. Mater. 4, 143 (2005).

${ }^{3}$ C. Y. Ng, T. P. Chen, M. S. Tse, V. S. W. Lim, S. Fung, and A. A. Tseng, Appl. Phys. Lett. 86, 152110 (2005), and references therein.

${ }^{4}$ G. Iannacone, A. Trellakis, and U. Ravaioli, J. Appl. Phys. 84, 5032 (1998).

${ }^{5}$ S. Das Sarma, R. de Sousa, X. Hu, and B. Koiller, Solid State Commun. 133, 737 (2005).

${ }^{6}$ T. Roschuk, J. Wojcik, E. A. Irving, M. Flynn, and P. Mascher, Proc. SPIE 5577, 450 (2004).

${ }^{7}$ F. Iacona, G. Franzo, and C. Spinella, J. Appl. Phys. 87, 1295 (2000).

${ }^{8}$ L. A. Nesbit, Appl. Phys. Lett. 46, 38 (1985).

${ }^{9}$ C. Bonafos et al., Nucl. Instrum. Methods Phys. Res. B 178, 17 (2001).
${ }^{10}$ F. Iacona, C. Bongiorno, C. Spinella, S. Boninelli, and F. Priolo, J. Appl. Phys. 95, 3723 (2004).

${ }^{11}$ M. Molinari, H. Rinnert, and M. Vergnat, Europhys. Lett. 66, 674 (2004).

${ }^{12}$ E. Fazio, E. Barletta, F. Barreca, F. Neri, and S. Trusso, J. Vac. Sci. Technol. B 23, 519 (2005).

${ }^{13}$ D. L. Williamson, Sol. Energy Mater. Sol. Cells 78, 41 (2003).

${ }^{14}$ J. I. Langford and A. J. C. Wilson, J. Appl. Crystallogr. 11, 102 (1978).

${ }^{15}$ H. G. Jiang, M. Ruhle, and E. J. Lavernia, J. Mater. Res. 14, 549 (1999)

${ }^{16}$ J. B. Pelka, W. Paszkowicz, P. Dluzewski, M. Brust, C. J. Kiely, M. Knapp, and E. Czerwosz, J. Alloys Compd. 328, 248 (2001).

${ }^{17}$ M. A. van Huis et al., Nucl. Instrum. Methods Phys. Res. B 191, 442 (2002).

${ }^{18}$ A. Weibel, R. Bouchet, F. Boulc'h, and P. Knauth, Chem. Mater. 17, 2378 (2005).

${ }^{19} \mathrm{X}$. Wu, Ch. Ossadnik, Ch. Eggs, S. Veprek, and F. Phillipp, J. Vac. Sci. Technol. B 20, 1368 (2002).

${ }^{20}$ P. H. Gaskell and D. J. Wallis, Phys. Rev. Lett. 76, 66 (1996).

${ }^{21}$ D. Comedi, O. H. Y. Zalloum, E. A. Irving, J. Wojcik, T. Roschuk, M. J. Flynn, and P. Mascher, Appl. Phys. Lett. (submitted).

${ }^{22}$ L. Levien, C. T. Prewitt, and D. J. Weidner, Am. Mineral. 65, 920 (1980).

${ }^{23}$ F. J. Himpsel, F. R. McFeely, A. Taleb-Ibrahimi, J. A. Yarmoff, and G. Hollinger, Phys. Rev. B 38, 6084 (1988).

${ }^{24}$ T. P. Chen, Y. Liu, C. Q. Sun, M. S. Tse, J. H. Hsieh, Y. Q. Fu, Y. C. Liu, and S. Fung, J. Phys. Chem. B 108, 16609 (2004).

${ }^{25}$ Note, in addition, that we deduce in some cases the presence of a (uniform) strain that shifts the $\mathrm{Si}$ (111) peak by few tenths of a degree [see Fig. 6(a)]. This effect is small as compared to the overall FWHM of the peak $\left(\sim 2^{\circ}\right)$.

${ }^{26}$ P. S. Prevey, Metals Handbook (American Society for Metals, Ohio, 1986), pp. 380-392.

${ }^{27}$ E. Lifshin, X-ray Characterization of Materials (Wiley-VCH, Weinheim, 1999).

${ }^{28}$ J. Pere, M. Gelize-Duvignau, and A. Lichanot, J. Phys.: Condens. Matter 11, 5827 (1999).

${ }^{29}$ D. Rafaja, V. Klemm, G. Schreiber, M. Knapp, and R. Kuzel, J. Appl. Crystallogr. 37, 613 (2004)).

${ }^{30}$ B. Fazio, M. Vulpio, C. Gerardi, Y. Liao, I. Crupi, S. Lombardo, S. Trusso, and F. Neri, J. Electrochem. Soc. 149, G376 (2002).

${ }^{31}$ X. Y. Chen, Y. F. Lu, L. J. Tang, Y. H. Wu, B. J. Cho, and X. J. Xu, J. Appl. Phys. 97, 014913 (2005).

${ }^{32}$ E. Fazio, E. Barletta, F. Barreca, F. Neri, and S. Trusso, J. Vac. Sci. Technol. B 23, 519 (2005).

${ }^{33}$ D. Tsoukalas, C. Tsamis, and P. Normand, J. Appl. Phys. 89, 7809 (2001).

${ }^{34}$ O. Jaoul, B. Bejina, F. Elie, and F. Abel, Phys. Rev. Lett. 74, 2038 (1995).

${ }^{35}$ G. Brebec, R. Seguin, C. Sella, J. Bevenot, and J. C. Martin, Acta Metall. 28, 327 (1980).

${ }^{36}$ T. Takahashi, S. Fukatzu, K. M. Itoh, M. Uematsu, A. Fujiwara, H. Kageshima, Y. Takahashi, and K. Shiraishi, J. Appl. Phys. 93, 3674 (2003).

${ }^{37}$ R. Kampmann and R. Wagner, Decomposition of Alloys: The Early Stages (Pergamon, Oxford, 1983), pp. 91-103.

${ }^{38}$ C. Spinella, S. Lombardo, and F. Priolo, J. Appl. Phys. 84, 5383 (1998).

${ }^{39}$ B. P. Uberuaga, M. Leskovar, A. P. Smith, H. Jonsson, and M. Olmstead, Phys. Rev. Lett. 84, 2441 (2000).

${ }^{40}$ J. M. Hyde, A. P. Sutton, J. R. G. Harris, A. Cerezo, and A. Gardiner, Modell. Simul. Mater. Sci. Eng. 4, 33 (1996). 